\title{
Molecular identification of emergent GII.P17-GII.17 norovirus genotype, Romania, 2015
}

S Dinu ${ }^{1}$, M Nagy ${ }^{2}$, DG Negru ${ }^{3}$, ED Popovici ${ }^{45}$, L Zota ${ }^{6}$, G Oprișan ${ }^{17}$

1. Molecular Epidemiology Laboratory, Cantacuzino National Institute of Research, Bucharest, Romania

2. 'Aurel Vlaicu' University of Arad, Arad, Romania

3. Arad Public Health Department, Arad Romania

4. Victor Babeș University of Medicine and Pharmacy Timișoara, Timișoara, Romania

5. Regional Centre of Public Health Timișoara, Timișoara, Romania

6. National Institute of Public Health - National Centre for Surveillance and Control of Communicable Diseases, Bucharest, Romania

7. Faculty of Pharmacy, Titu Maiorescu University, Bucharest, Romania

Correspondence: Sorin Dinu (sorind@cantacuzino.ro)

Citation style for this article:

Dinu S, Nagy M, Negru D, Popovici E, Zota L, Oprișan G. Molecular identification of emergent GII.P17-GIl.17 norovirus genotype, Romania, 2015. Euro Surveill. 2016;21(7): pii=30141. DOI: http://dx.doi.org/10.2807/1560-7917.ES.2016.21.7.30141

Article submitted on 02 February 2016 / accepted on 18 February 2016 / published on 18 February 2016

The novel GII.P17-GII.17 norovirus genotype has been reported as cause of gastroenteritis outbreaks in China and Japan since the winter season 2014/15, replacing the pandemic strain GII.4 Sydney 2012. These emergent strains have also been sporadically reported on other continents than Asia. GII.P17-GII.17 isolates, similar to Kawasaki308 2015, were identified in three patients during a large outbreak of acute gastroenteritis affecting 328 people in Romania, in neighbouring localities, in 2015.

We present molecular evidence for the circulation of emergent norovirus GII.P17-GII.17 strain during an outbreak of acute gastroenteritis that occurred in Arad, a county in the western part of Romania, in 2015.

Noroviruses are among the leading causes of non-bacterial gastroenteritis worldwide [1]. Published data on circulation of noroviruses in Romania are scarce $[2,3]$ and sequences previously obtained by our group (i.e. GenBank FR695414-FR695417) indicated circulation of genotype GII.P21-GII.2 in 2006 (unpublished data).

\section{Origin of samples}

Between 16 October and 1 December 2015, an outbreak of acute gastroenteritis was recorded in Arad, a county in the western part of Romania, bordering Hungary, with ca 400,000 inhabitants. The first cases were notified by a local hospital to Arad Public Health Department on 18 October 2015. The public health authorities further investigated the outbreak-associated cases among household contacts, in schools and hospitals. Suspected cases were defined as patients with three or more loose stools in a 24 -hour period, and/or two or more episodes of vomiting in a 24 -hour period. Confirmed cases were suspected cases additionally testing positive for norovirus by reverse transcription-polymerase chain reaction (RT-PCR). Three hundred twenty-eight cases (sex ratio: 0.8:1; 145 male: 183 female) were recorded across 20 small rural and urban neighbouring communities with a total number of ca 35,000 inhabitants. The patients' median age was 18 years and the average age was 27 years (standard deviation: 22; interquartile range: 10-40; age range: $0-95$ years). Cases were either clustered in foci $(n=302)$ or sporadic $(n=26)$. One hundred fourteen cases were recorded in schools. The illness was mild, self-limiting, with only eight patients hospitalised. No further information was available on the reasons for hospitalisation. No fatalities were recorded. The onset was sudden and symptoms included nausea $(n=220)$, vomiting $(n=214)$, abdominal pain $(n=178)$, diarrhoea $(n=168)$, headache $(n=100)$, dehydration $(n=30)$ and fever $(n=20)$. The index case was not identified.

\section{Laboratory investigation}

Five stool samples collected between 25 October and 7 November 2015 from patients with acute gastroenteritis were received for molecular diagnostic and genotyping at Cantacuzino National Institute of Research in Bucharest.

Viral RNA was extracted from $140 \mu \mathrm{L}$ of PBS stool suspension (10\% wt/vol) using QIAamp RNA Viral Mini Kit (Qiagen). A fragment of 1111 nt spanning ORF1-ORF2 (RdRp-VP1) junction, region recommended for norovirus typing, was amplified using primers JV12 and G2SKR $[4,5]$.

Three out of five samples yielded a PCR product suitable for sequencing (one sample from an adult and two samples from two young children). Sequencing was performed with BigDye Terminator v3.1 (Applied Biosystems) and Norovirus Genotyping Tool (http:// 
Neighbour-joining tree of GII norovirus isolates based on partial RdRp-VP1 junction, outbreak of acute gastroenteritis, Romania, 2015
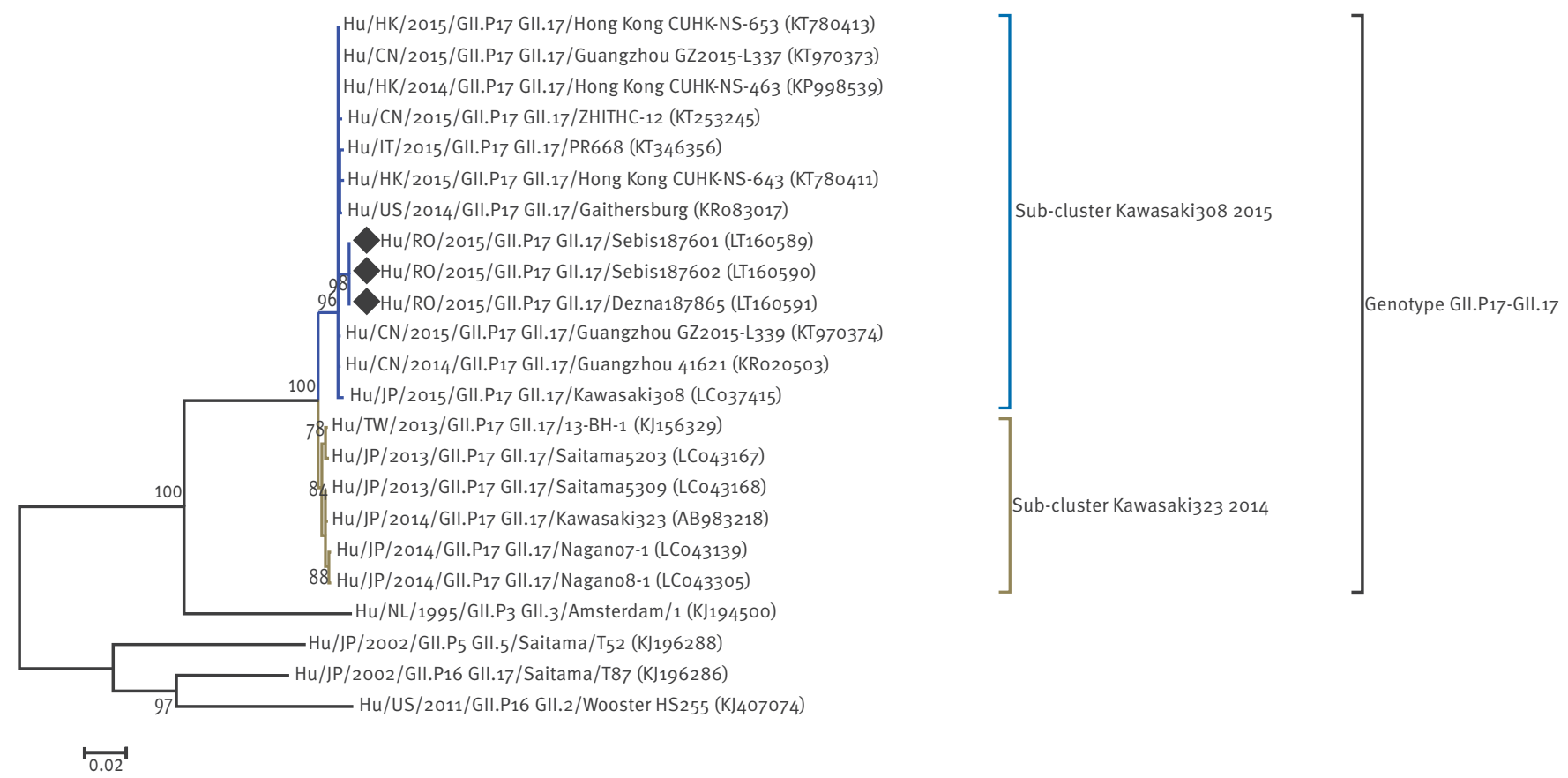

Black diamonds: sequences obtained in this study from three patients during an outbreak of acute gastroenteritis in Arad county, Romania, 2015.

Numbers at nodes represent the bootstrap percentages (values $<70 \%$ are not shown). The analysis was conducted on a 1067 nt sequence (nt positions 4303-5369 in isolate Kawasaki323 2014, GenBank accession number AB983218).

www.rivm.nl/mpf/norovirus/typingtool) was employed for assigning genotype [6]. The three sequences obtained were identical and the results of typing indicated genotype GII.P17-GII.17. Sequences were deposited in GenBank under the accession numbers LT160589, LT160590, and LT160591.

Phylogenetic analysis was conducted with Mega 6 software [7], neighbour-joining statistical method, Kimura 2-parameter, 1,000 bootstrap replications. Phylogenetic analysis (Figure) grouped the sequences described here in the same sub-cluster with Kawasakizo8 strain, identified in Japan in 2015 [8].

\section{Discussion}

Gll.17 norovirus genotype was first described in French Guiana, in 1978 [9]. As reviewed elsewhere [10], GII.17 strains have been sporadically detected in Africa, America, Asia and Europe. Strains belonging to the novel GII.P17-GII.17 norovirus genotype were detected in Korea in 2013 and have been associated with gastroenteritis outbreaks in China and Japan since 2014/15 winter season, replacing the previously dominant strain GIl.4 Sydney 2012 [8,10-13]. The same genotype was identified in groundwater in Kenya, in 2012 [14]. Molecular dating analysis estimated that GII.P17-GII.17 strains have been circulating in Asia as early as 2002 [8].
In Europe, GII.P17-GII.17 strains were found in sporadic cases from France (2013), Italy (2015), the Netherlands and Russia $[10,15]$. The only study providing a detailed molecular characterisation of a GII.P17-GII.17 norovirus strain detected in Europe comes from Italy. There, the strain was identified in two sporadic cases of acute severe gastroenteritis occurring in February 2015 among young children residing in two distinct Italian regions [15].

GII.P17-GIl.17 strains encode a new type of RNAdependent RNA polymerase and the VP1 capsid protein displays amino acid substitutions in major epitopes. Also, the emerging GII.P17-GIl.17 genotype is undergoing a fast diversification which led to two subclusters based on RdRp and capsid genes. The two sub-clusters are represented by Kawasaki323 2014, and Kawasaki3o8 2015 strains, respectively [8]. As demonstrated for Gll.4 genotype strains, mutations in VP1 can lead to evasion from host immune system [16].

We report here the detection of GII.P17-GII.17 norovirus isolates highly related to Kawasakizo8 strain (99.15\% nt sequence similarity in the analysed fragment), identified in Japan in 2015. The sequences described were obtained during an outbreak of acute gastroenteritis in late 2015 in the western part of Romania and were identified in two young children (under five years) and in a young adult (under 30 years) with acute gastroenteritis. 
The patients resided in two neighbouring localities. Our molecular analysis based on a DNA fragment of the ORF1-ORF2 (RdRp-VP1) junction (Figure), indicates that the Romanian strains belong to Kawasakizo8 2015 sub-cluster (100\% bootstrap value). This sub-cluster comprises strains identified in 2014 and 2015 in Hong Kong and in the city of Guangzhou, Guangdong province, China. Gastroenteritis outbreaks caused by this emergent genotype were recorded in the provinces of Jiangsu and Guangdong in China, in 2014 and 2015 $[11,12]$. Also, the Italian strain [15] and an isolate identified in 2014 in a sporadic case of acute gastroenteritis in a three-year-old child from the United States [17] are closely related to the Romanian isolates.

\section{Conclusion}

This documented outbreak, outside Asia, was caused by a norovirus strain belonging to the emergent GII. P17-GIl.17 genotype which in the near future might become the dominant genotype in Europe. Due to the limited number of diagnosed cases, we cannot totally exclude the possibility that this gastroenteritis outbreak could have been caused by another pathogen; however, considering the clustering in time and place and rapid onset of symptoms, norovirus was the most probable cause of this outbreak. Therefore, national and European surveillance systems should be prepared for events associated to this emergent strain.

\section{Acknowledgements}

The authors are grateful to $\mathrm{Dr}$ Codruța-Romanița Usein (Cantacuzino National Institute of Research, Bucharest, Romania) for her kind support during this study and for critical review of the manuscript.

\section{Conflict of interest}

None declared.

\section{Authors' contributions}

MN, DGN and EDP collected and analysed epidemiological data. LZ managed the acute diarrhoea syndrome surveillance programme. SD and GO performed sequencing and phylogenetic analysis. SD wrote the paper. GO revised the manuscript.

\section{References}

1. Atmar RL, Estes MK. The epidemiologic and clinical importance of norovirus infection.Gastroenterol Clin North Am. 2006;35(2):275-90, viii. DOI: 10.1016/j.gtc.2006.03.001 PMID: 16880066

2. Damian M, Tatu-Chițoiu D, Usein CR, Oprişan G, Palade AM, Dinu S, et al. Laboratory diagnosis of infectious diarrhoea syndrome; a three years study in two hospitals of infectious diseases. Roum Arch Microbiol Immunol. 2009;68(2):89-94. PMID: 20361527

3. Spina A, Kerr KG, Cormican M, Barbut F, Eigentler A, Zerva L, et al. Spectrum of enteropathogens detected by the FilmArray GI Panel in a multicentre study of community-acquired gastroenteritis. Clin Microbiol Infect. 2015;21(8):719-28. DOI: 10.1016/j.cmi.2015.04.007 PMID: 25908431

4. Kojima S, Kageyama T, Fukushi S, Hoshino FB, Shinohara M, Uchida K, et al. Genogroup-specific PCR primers for detection of Norwalk-like viruses. J Virol Methods. 2002;100(1-2):107-14. DOI: 10.1016/S0166-0934(01)00404-9 PMID: 11742657

5. Vinjé J, Koopmans MP. Molecular detection and epidemiology of small round-structured viruses in outbreaks of gastroenteritis in the Netherlands.J Infect Dis. 1996;174(3):6105. DOI: 10.1093/infdis/174.3.610 PMID: 8769621

6. Kroneman A, Vennema H, Deforche K, v d Avoort H, Peñaranda $\mathrm{S}$, Oberste MS, et al. An automated genotyping tool for enteroviruses and noroviruses. J Clin Virol. 2011;51(2):121-5. DOI: 10.1016/j.jcv.2011.03.006 PMID: 21514213

7. Tamura K, Stecher G, Peterson D, Filipski A, Kumar S. MEGA6: Molecular Evolutionary Genetics Analysis version 6.0. Mol Bio Evol. 2013;30(12):2725-9. DOI: 10.1093/molbev/mst197 PMID: 24132122

8. Matsushima Y, Ishikawa M, Shimizu T, Komane A, Kasuo $S$, Shinohara M, et al. Genetic analyses of GII.17 norovirus strains in diarrheal disease outbreaks from December 2014 to March 2015 in Japan reveal a novel polymerase sequence and amino acid substitutions in the capsid region. Euro Surveill. 2015;20(26):21173. DOI: 10.2807/1560-7917. ES2015.20.26.21173 PMID: 26159307

9. Rackoff LA, Bok K, Green KY, Kapikian AZ. Epidemiology and evolution of rotaviruses and noroviruses from an archival WHO Global Study in Children (1976-79) with implications for vaccine design.PLoS One. 2013;8(3):e59394. DOI: 10.1371/journal. pone.0059394 PMID: 23536875

10. de Graaf $M$, van Beek J, Vennema H, Podkolzin AT, Hewitt J, Bucardo F, et al. Emergence of a novel Gll.17 norovirus End of the GII.4 era? Euro Surveill. 2015;20(26):21178. DOI: 10.2807/1560-7917.ES2015.20.26.21178 PMID: 26159308

11. Fu J, Ai J, Jin M, Jiang C, Zhang J, Shi C, et al. Emergence of a new GII.17 norovirus variant in patients with acute gastroenteritis in Jiangsu, China, September 2014 to March 2015. Euro Surveill. 2015;20(24):21157. DOI: 10.2807/15607917.ES2015.20.24.21157 PMID: 26111236

12. Lu J, Sun L, Fang L, Yang F, Mo Y, Lao J, et al. Gastroenteritis Outbreaks Caused by Norovirus GII.17, Guangdong Province, China, 2014-2015. Emerg Infect Dis. 2015;21(7):1240-2. DOI: 10.3201/eid2107.150226 PMID: 26080037

13. Kim JS, Kim HS, Hyun J, Song W. Molecular Epidemiology of Human Norovirus in Korea in 2013. Biomed Res Int. 2015;2015:468304.

14. Kiulia NM, Mans J, Mwenda JM, Taylor MB. Norovirus GII.17 Predominates in Selected Surface Water Sources in Kenya. Food Environ Virol. 2014;6(4):221-31. DOI: 10.1007/s12560-0149160-6 PMID: 25059212

15. Medici MC, Tummolo F, Calderaro A, Chironna M, Giammanco GM, De Grazia S, et al. Identification of the novel Kawasaki 2014 Gll.17 human norovirus strain in Italy, 2015. Euro Surveill. 2015;20(35):30010. DOI: 10.2807/1560-7917. ES.2015.20.35.30010 PMID: 26530698

16. Debbink K, Lindesmith LC, Donaldson EF, Baric RS Norovirus immunity and the great escape.PLoS Pathog. 2012;8(10):e1002921. DOI: 10.1371/journal.ppat.1002921 PMID: 23093932

17. Parra GI, Green KY. Genome of Emerging Norovirus GII.17, United States, 2014. Emerg Infect Dis. 2015;21(8):1477-9. DOI: 10.3201/eid2108.150652 PMID: 26196235

\section{License and copyright}

This is an open-access article distributed under the terms of the Creative Commons Attribution (CC BY 4.0) Licence. You may share and adapt the material, but must give appropriate credit to the source, provide a link to the licence, and indicate if changes were made.

This article is copyright of the authors, 2016. 